\title{
Spotlight on dream recall: the ages of dreams
}

\section{Anastasia Mangiaruga \\ Serena Scarpelli \\ Chiara Bartolacci \\ Luigi De Gennaro}

Department of Psychology, University of Rome "Sapienza", Rome, Italy
Correspondence: Luigi De Gennaro

Department of Psychology, University of Rome "Sapienza", Via dei Marsi,

78, 00185 Rome, Italy

Tel +3906 499| 7647

Fax +3906 4991 771।

Email luigi.degennaro@uniroma l.it
This article was published in the following Dove Press journal: Nature and Science of Sleep

\begin{abstract}
Brain and sleep maturation covary across different stages of life. At the same time, dream generation and dream recall are intrinsically dependent on the development of neural systems. The aim of this paper is to review the existing studies about dreaming in infancy, adulthood, and the elderly stage of life, assessing whether dream mentation may reflect changes of the underlying cerebral activity and cognitive processes. It should be mentioned that some evidence from childhood investigations, albeit still weak and contrasting, revealed a certain correlation between cognitive skills and specific features of dream reports. In this respect, infantile amnesia, confabulatory reports, dream-reality discerning, and limitation in language production and emotional comprehension should be considered as important confounding factors. Differently, growing evidence in adults suggests that the neurophysiological mechanisms underlying the encoding and retrieval of episodic memories may remain the same across different states of consciousness. More directly, some studies on adults point to shared neural mechanisms between waking cognition and corresponding dream features. A general decline in the dream recall frequency is commonly reported in the elderly, and it is explained in terms of a diminished interest in dreaming and in its emotional salience. Although empirical evidence is not yet available, an alternative hypothesis associates this reduction to an age-related cognitive decline. The state of the art of the existing knowledge is partially due to the variety of methods used to investigate dream experience. Very few studies in elderly and no investigations in childhood have been performed to understand whether dream recall is related to specific electrophysiological pattern at different ages. Most of all, the lack of longitudinal psychophysiological studies seems to be the main issue. As a main message, we suggest that future longitudinal studies should collect dream reports upon awakening from different sleep states and include neurobiological measures
\end{abstract} with cognitive performances.

Keywords: dreaming, sleep mentation, development, sleep, continuity hypothesis, aging

\section{Introduction}

Several issues still puzzle researchers who try to define the qualitative and quantitative features of dream production. The most promising approach highlights the neurobiological basis and cognitive mechanisms involved in dream experience. Considering brain sleep covarying maturation, from fetal development to early childhood, going through adulthood and elderly, some authors have hypothesized a role for dream content and dream recall-related neural correlates in the development of cognitive processes.$^{1-7}$ In this paper, we will review the available evidence on changes in dream recall across the life span, focusing mainly on a cognitive and neurobiological level, and try to pinpoint its complexity and hypothetical role in overall mental development 
(ie, at a cognitive, emotional, and neural levels). We will try to address how the neurobiological and psychological understanding of the ontogeny of sleep mentation contributes to our understanding of the cognitive and emotional development of a human being.

One of the most recognized explanatory hypotheses of dreaming is the so-called "continuity hypothesis" between waking and sleep mental functioning. First proposed by Bell and Hall in $1971^{8,9}$ as the recurrence of thematic content from daily experience in the dreams of their patients, it aimed to study dream recall as a direct psychological correlate of personality and emotional features of daily mental activity. ${ }^{8-12}$ From a different perspective, the hypothesis posits a "continuum" between dreaming and neurobiological and cognitive functioning. ${ }^{12-14}$ It should be underlined that these two viewpoints are not mutually exclusive, and we will consider both perspectives in our review.

In dream research, the methodological limitation to reliably access the mental sleep activity (ie, the impossibility to directly observe what is happening in our minds) $)^{15,16}$ makes the definition of dreaming difficult. The scientists interested in dream experience refer to "dream generation" as the precise moment in which dreams are shaped (producing the "sleep mentation"). ${ }^{15,16}$ Furthermore, the dream recall studies are directly focused on the retrieval of dream contents upon the awakenings, considering dream recall as a peculiar form of episodic-declarative memory.

The very beginning of a systematic study of dreaming traditionally refers to the discovery of rapid eye movement (REM) sleep ${ }^{15-19}$ and the consequent notion that sleep mentation is a phenomenon restricted to that physiological state. This correspondence has been subsequently confuted by Foulkes, ${ }^{20}$ who found that sleep mentation in non-rapid eye movement (NREM) sleep simply changes the way we approach it, by defining it not just as story-like contents but as "anything passing through your mind". ${ }^{20}$ This precaution led to the discovery of presence of sleep mentation also in NREM sleep, opening new perspectives in methods and theories about dream experience analysis and its neural correlates. ${ }^{25-28}$ Moreover, Solms ${ }^{25,26}$ in a review of cases concerning global or partial cessation of dreaming subsequent to the brain lesions (termed as "anoneria") found no direct relationship between REM sleep and dream recall. Specifically, no correlation was found between lesions in brainstem regions that modulate REM sleep generation and dream recall cessation, while focal cortical lesions can result in dream recall disappearing in a context of still preserved REM sleep production. ${ }^{25}$ As a result, different theoretical approaches have been developed in the attempt to define the electroencephalographic (EEG) correlates of dream recall. ${ }^{20-26}$ Still far from solving the problem, most investigators moved the dichotomy from REM/NREM sleep mentation to the qualitative difference of dream report in each sleep stage. ${ }^{32} \mathrm{~A}$ basic limitation of these models is to assume dream content to be generated at the same stage upon which the subject has been awakened, while the transition from sleep to wakefulness is associated to physiological changes that make understanding the relationship between dream content and its neural correlates difficult. ${ }^{20,21}$ To overcome this perspective, dream studies have been focused on the search for a parallelism between psychophysiological and neural correlates of dream recall and declarative memory processes, its encoding, and recollection. ${ }^{21,27-35}$ As a result, sleep mentation has been further evaluated by analyzing the last segment of the EEG activity in correspondence with provoked awakenings, to associate EEG patterns and dream recall occurrence. ${ }^{26-36}$

\section{Tools and trends in dream research}

Bearing in mind that dream experience is a peculiar object of study, it is necessary to take into account the limitations of the different methods used in dream research.

Investigations on dream content usually use three different self-report techniques: retrospective questionnaires, dream diaries, and prospective reports. ${ }^{37-39}$ Retrospective methods collect past experiences of dreaming spanning periods of variable length (weeks, months, or years). ${ }^{39,40}$ Nonetheless the advantage of quick data collection from large samples and with low expenses, these methods have a strong memory bias due to the possible influence of everyday activity in the retrieving of quantitative and qualitative dream features. ${ }^{38}$ Prospective methods concern daily diaries for a short period or, less frequently, a written report of any dream recalled. ${ }^{39}$ Its advantage consists of an improvement in dream recall rates and more detailed reports, ${ }^{37}$ allowing longitudinal studies. It also is less influenced by memory biases. ${ }^{37-41}$

Similarly, provoked awakenings associated with dream report collection partly overcome most of the known biases in memory recollection. Moreover, this strategy allows reliable time coupling with polysomnographic (PSG) recordings, with consequent advantages due to the possibility to control over the time of recollection and the sleep stage of awakening. For these reasons, it remains the gold standard in dreams studies. ${ }^{37,41,42}$ However, sleep laboratory studies may affect dream contents, as can be observed by the frequent incorporation of the experimental setting. ${ }^{41}$ For this reason, it has been suggested to consider the known "first night effect" not just 
for what concerns the influence on sleep quality but also for a control over the quality of dream report. ${ }^{43}$

For what concerns the qualitative features of dreams, the most common method is the coding system developed by Hall and van de Castle in $1966^{44}$ in its recent revisions and updates, ${ }^{45-47}$ which systematically investigates some features of dream content (ie, characters, social interactions and activities, success and failure, misfortune and good fortune, emotions, settings, objects, and descriptive elements) through categories and Likert scales. ${ }^{44-47}$ Additionally, these methods permit to look for the memory sources of dreams, searching for a correspondence between dream reports and contents recorded by the subjects in a diary of daytime events. ${ }^{48-51}$ In other words, some dream experiences could incorporate waking events. ${ }^{48-51}$

Dream features, as well as quantitative features of sleep, undergo the influence of circadian rhythms. ${ }^{52-57}$ For this reason, multiple nighttime awakening methods and daytime nap protocols have been developed to understand how dream content, as well as EEG patterns, are influenced by the sleep stage or by the time of the day. ${ }^{58,59}$

For what concern the morning awakenings, some investigations revealed that dream report features collected after a night of uninterrupted sleep at home are not dissimilar from those collected after night protocol in laboratory. ${ }^{60,61}$ Furthermore, it should be noted that circadian and ultradian variations could affect the characteristics of dream reports. ${ }^{62}$ In this respect, qualitative features of dream experiences reported from NREM and REM awakenings in the second half of the night are similar and more "dreamlike". Conversely, dream contents reported from REM and NREM sleep stages at the beginning of the night show different qualitative features (ie, NREM dream reports are more "thought-like" than REM dream reports). ${ }^{63-65}$

All these aspects must be taken into account for a complete comprehension and discussion about dreaming along different protocols.

\section{Dreaming in childhood}

Dream studies in infancy have been rarely focused on healthy subjects, being usually a report of bad dream frequency and dream content analysis in parasomnias or psychiatric conditions. ${ }^{66-70}$

In the contest of systematic investigations about healthy cohorts, Foulkes ${ }^{2-4}$ was one of the firsts to investigate dream content in children. In a laboratory study, he found dream frequency rates of $20 \%$ for children younger than 7 years, as compared to $80 \%-90 \%$ frequency rate in adults. ${ }^{4}$ These results partly overlap latest investigations about home dream recall frequency in children compared to a group of young adults, in which the frequency of home dream by retrospective questionnaires was significantly lower in children. ${ }^{71}$

In an attempt to investigate the neurobiological mechanisms involved in dreaming, Foulkes ${ }^{2-4}$ explored the hypothesis that dream recall is directly reflected by cognitive maturation, in a correlational study between sleep mentation and performances in some neuropsychological tests. ${ }^{2-4}$ Bearing in mind the impossibility to collect detailed dream reports in children below 3 years old, due to the so-called "infantile amnesia" (namely, the impossibility to report memories due to not yet developed memory neural systems),${ }^{72-74}$ he started from the assumption of a general limitation in language production and emotional comprehension as the key problem of a dream recall report in infancy. ${ }^{75}$ More interestingly, while no correlation with language development measures was found, ${ }^{2-4}$ the best predictor of dream recall was the Block Design Test (subtest of the Weschsler Intelligence Scale for Children [WISC] $)^{76}$ which is known to be a reliable measure of visuospatial and organizational skills. ${ }^{76,77}$ The dependent variables in Block Design Test are usually accuracy and speed in completing each item. ${ }^{76,77}$ In particular, the subjects were asked to use hand movements to rearrange blocks, trying to match with a specific configuration. ${ }^{76,77}$

Due to these findings, the study of dream recall rates in children moved from a language-emotional level to an assessment of executive functions, with empirical evidence in favor of a correlation between mental imagery and sleep mentation. ${ }^{4-6,69}$

A renewed interest in dream content in childhood recently arose from studies measuring different cognitive skills in early childhood..$^{67,67,68,78}$ Specifically, Sàndor et al ${ }^{6,7}$ assessed the hypothesis of a relationship between executive abilities and dream content across development. The authors assessed the executive attention development in a group of 40 children from 4 to 8.5 years of age, administering the Stroop Test, the Emotional Stroop Test, and the Child Attention Network Test. ${ }^{7}$ They found a positive association between the number of characters per dream and the selection of relevant stimuli in a distracting environment (Orienting Network). The number of self-initiated actions and the ratio of gross motor activities in dreams were significantly associated with the Incongruency Index of the Stroop Test. ${ }^{7}$ Gross motor activities were associated with higher accuracy in the condition of incongruent stimuli at the Stroop Test. Both results were interpreted in terms of more efficient behavioral inhibitory control with more dreamer-involved actions. Additionally, 
the number of verbal actions per dream correlated positively with the Vocabulary Subtest of the WISC-IV. ${ }^{7}$ Dreamerinitiated interactions per dream were associated with the behavioral inhibitory control functions, as measured by the Incongruency Index of the Stroop Test. ${ }^{7}$ Friendly interactions per dream showed an association with the Emotional Interference Index of the Stroop Test, correlating positively with a more efficient control of emotional interference. ${ }^{7}$ The general results point to a positive association between dream features and a better control over executive functions. In contrast with Foulkes' study, ${ }^{2-4}$ no correlation between visuospatial abilities (measured through the Block Design Subset - WISC-IV) $)^{76,77}$ and the number of recalled dreams has been found, even when a possible moderating effect of age was controlled. Also, Sàndor et $\mathrm{al}^{7}$ did not find a correlation between dream bizarreness and some neuropsychological measures, partially in disagreement with previous findings by Colace, ${ }^{79}$ who found a correlation between long-term memory and the bizarreness of dreams in children between 3 and 5 years of age. ${ }^{79}$

Another perspective for studying sleep mentation in childhood is the general analysis of incidence of bad dreams. ${ }^{40,67-69}$ From studies about dream recall in pathological conditions, such as recurrent nightmares, post-traumatic stress disorder (PTSD), and severe trauma, empirical studies have shown an increased dream recall rates compared to healthy cohort, though no univocal explanation about the underlying mechanisms is available so far. ${ }^{40,66-70}$

Dream recall rates in traumatized children have been also investigated. ${ }^{80-84}$ Studies in children living in conditions of war and military violence are in line with previous findings. Specifically, an association between stressful events and higher dream recall rate has been found in a group of Palestinian children aged 6-16 years who lived in a traumatized environment, compared to nontraumatized children. ${ }^{84}$ The authors found enhanced dream recall and more contextual coherent images in dream content. ${ }^{84}$ In addition, in children who suffered of PTSD because of a kidnap, the more the children had ability to verbalize their feelings, the more they dreamed about the trauma. ${ }^{83}$ This is consistent with studies on verbal ability and dream recall frequency. ${ }^{4-7,67-69,75,83}$

When applied to memory for dreams, children with PTSD who have more unpleasant and traumatic dreams may have heightened dream recall because of the emotionally salient nature of their dreams. ${ }^{67-93}$ These findings were interpreted in terms of a general sleep disruption and subsequent increased possibility of a post-nightmare awakening, which leads to increased dream recall. ${ }^{84}$
The modification of memory performance across early childhood, due in the firsts ages to the so-called infantile amnesia $^{72-74}$ and subsequently to the development of cognitive functions, ${ }^{67}$ parallel the improvement of dream recall rates in children.

Nonetheless dream content and dream recall studies have been made both on healthy cohort and pathological samples at any age, there is no agreement about dream content qualitative features and dream recall rates in the life span among age groups. ${ }^{5,67-69,72}$

This lack of a consistent literature about dream recall across ages that goes from early childhood to first adolescence is partly due to the difficulty in collecting reliable dream report from children. A low compliance in recording dream content may be due to the presence of a stranger (ie, the researcher) and can lead to a bias in reporting dreams. ${ }^{5-7}$ Furthermore, dream reports in children are often collected in absence of EEG monitoring, considered the gold standard for a reliable time coupling between defined sleep stage awakenings and dream reports. ${ }^{27}$ Consequently, the most used method in children's dream research is the exclusive collection of dream reports. ${ }^{5,67-69}$ On one hand, it should be considered that the content analysis methods are widely defined in literature, ${ }^{16,44,45,85-88}$ while, on the other hand, the presence of different methods for collecting dream reports in children leads to additional biases, when a comparison with content analysis in adulthood is requested. ${ }^{69}$ In fact, dream reports are more often collected at home by a caregiver instead of being reported to the researcher or directly by the child, which potentially misleads the actual dream content. ${ }^{69}$

Interestingly, another issue in facing dream research in infancy is the achievement of the ability to discern a dream from reality, whose age definition is still controversial. 4,69,73,89,90 The ages in which children begin to report some dream content seem to parallel their cognitive maturation. ${ }^{73}$ Some empirical evidence showed that children start at 4 years of age to discern dream from reality and reach a complete comprehension of this difference at the age of 9 years, when cognitive abilities are adequately matured. ${ }^{4,69,73,89,90}$ At the same time, dream content analysis reveals a full development and adult-like content of dream experience from 9 years of age, ${ }^{4,89,73,91}$ coherent with mature mental imagery skills. ${ }^{73,92}$ Specifically, dream content at the age of 2-5 years shows a general absence of people, object, and events, being usually a static report of single characters. Longer reports, but with still low recall rate, are reached between ages 5 and 7 years. A first real development in dream content is reached at the age of 7 years, when the child starts to represent himself in 
the dream, with higher emotional content, active participation, and narrative structure, in dreams overall more frequent and longer. ${ }^{4,73,89,91}$

Considering these incongruences and the difficulty to bring together the few findings about dream production in children, we can only claim for further investigations about neuropsychological measures, ${ }^{26}$ EEG waking mental activity, ${ }^{94}$ and the EEG correlates of dreaming, with an aim to deepen the knowledge about the role of sleep mentation in brain maturation, for a better comparison with qualitative aspect of dream experience in future.

\section{Dreaming in adulthood}

A direct comparison between dream experience in infancy and adulthood cannot be addressed, due to the lack of studies focused on this topic. Actually, PSG recordings with provoked awakenings are the most commonly used method to investigate dream recall in adulthood. For this reason, we will mostly discuss the electrophysiology of dreaming while reviewing studies in this age range.

Studies about brain rhythms in wakefulness indirectly highlight the possible background of development in sleep mentation. The alpha $(\sim 8.00-11.75 \mathrm{~Hz})$ and theta $(\sim 5.00$ $7.75 \mathrm{~Hz}$ ) rhythms seem to be pivotal activities both in brain maturation $^{94}$ and in a successful dream recall. ${ }^{27,28}$

Brain maturation has been linked to changes in the alpha and theta activity across the life span. ${ }^{94}$ Specifically, a progressive increase of the alpha activity in its absolute power has been observed from early childhood until the first adolescence, with a contemporary reduction in the absolute delta $(\sim 1.5-3.5 \mathrm{~Hz})$ and theta power. ${ }^{93}$ In infancy, the alpha rhythm maturation follows a posterior-anterior gradient in wakefulness. ${ }^{94}$ This trend is mirrored during sleep onset in children between the ages of 0 and 48 months, ${ }^{94}$ gaining attention due to the relevance of EEG rhythms in posterior areas for a successful dream recall in adulthood ${ }^{28}$ Additionally, the passage from resting states to cognitive performance in wakefulness is epitomized by the alpha rhythm suppression and by the shift to a prevalent theta activity. ${ }^{94}$ The topographical changes of cortical oscillatory activity during sleep involve regions upon which relies a successful memory encoding in wakefulness. ${ }^{96}$ Namely, from a neuropsychological to an electrophysiological level, dream recall seems to rely on mechanisms comparable to episodic-declarative memory functioning. ${ }^{15,21}$ The theta activity in its medial frontal gradient, also known as "frontal midline theta", is involved in memory processes ${ }^{29}$ and is considered predictive of cognitive performance while awake. ${ }^{33,97}$ Additionally, the theta activity has been found to be a fundamental property of hippocampus-prefrontal cortex connectivity. ${ }^{98}$ Furthermore, Norman et al ${ }^{99}$ suggested its role as a suppressor of the external interference with selective retrieval of target memory during cognitive tasks or in meditative state, and Domhoff ${ }^{11}$ addressed its importance in the so-called "default mode network" functioning. In this context, common mechanisms in the processes underlying encoding and recall of episodic memories across different states of consciousness have been suggested. ${ }^{21}$

Recent findings by Scarpelli et $\mathrm{al}^{21,27,35}$ pointed to a statedependent EEG background for a successful dream recall. This confirmed the pivotal importance in defining the electrophysiology of sleep mentation to solve the controversies of a functional (and developmental) meaning of dream experience. For what concerns the specific EEG patterns of dream recall, both Esposito et $\mathrm{al}^{31}$ and Marzano et $\mathrm{al}^{28}$ observed that a decreased temporoparietal alpha during NREM sleep is predictive of subsequent dream recall, while the theta oscillations in the last 5 minutes of REM sleep predict the subsequent dream recall both during nap ${ }^{27}$ and nighttime ${ }^{28}$ investigations. This seems also coherent with the reported relationship between the prefrontal theta activity during REM sleep and the significant positive consolidation of negative vs neutral emotional memories in a nap protocol. ${ }^{96}$

Recent findings addressed the existence of a "hot zone" (ie, parietal occipital areas), whose low-frequency (1-4 $\mathrm{Hz}$ ) decrease was predictive of dream recall, after both REM and NREM awakenings. ${ }^{100}$ Consequently, a successful dream recall was considered dependent on the state of EEG activation. ${ }^{100}$ Similarly, a previous within-subjects study had revealed that brain activity shows higher power in high-frequency range (gamma activity: $18-50 \mathrm{~Hz}$ ), which is associated to the presence of recall on awakening both during REM and NREM sleep. ${ }^{59}$

Despite the dichotomy, REM sleep dreaming is considered outdated; very few explanations on the encoding and retrieval of dream contents from NREM sleep have been proposed. In one of those, Scarpelli et $\mathrm{al}^{35}$ again found a decreased delta power in temporo-parietal regions prior to a successful compared to an unsuccessful dream recall after NREM awakenings in a multiple nap protocol. ${ }^{35}$ This is coherent with a decline of the delta and sigma EEG power over the centro-parietal regions in dream report from NREM sleep. ${ }^{101}$ Augmented slow-wave sleep (SWS), together with a longer total sleep time and lower intrasleep awakenings in the recovery sleep after sleep deprivation, almost completely abolishes dream recall. ${ }^{86}$ Moreover, an increased dream recall frequency has been observed in insomniacs, whose sleep is 
characterized by a raise in fast-frequency EEG activity, ${ }^{102}$ as well as in normal sleeper with higher number of intrasleep wakefulness. ${ }^{103,104}$ In the same vein, the stimulation on the fronto-temporal area in the gamma band by transcranial direct current stimulation during REM sleep elicits lucid dreams. ${ }^{105}$

However, there are still confounding findings that cannot lead to a univocal explanation of EEG activity and presence/ absence of dreaming. Some studies reported a relationship between high-frequency activity and dream recall. ${ }^{59,105}$ Some of these findings are consistent with the hypothesis that an increased brain activity is necessary for the retrieval of sleep mentation. ${ }^{52,106-108}$ Therefore, it has been suggested that EEG activation could facilitate the consolidation and storage of the mental sleep contents during sleep. ${ }^{35}$

For what concern the qualitative features of dream reports, dream bizarreness has been linked to cognitive processes and skills, not only in developmental but also in adult studies. ${ }^{92,109}$ Interestingly, dream bizarreness was negatively correlated with right hippocampus and left amygdala volumetry in healthy subjects. ${ }^{87}$ At the same time, lower bizarreness and lower emotional load has been found in Parkinson's disease (PD) patients with a hypodopaminergic state. Visual vividness has been found to positively correlate with the volume of amygdala and the thickness of the left medial prefrontal cortex (mPFC) in PD patients, while emotional load is positively correlated with hippocampal volume, suggesting a role of amygdala-mPFC dopaminergic network system in dream recall. ${ }^{88}$ This partly supports the hypothesis of a role of paralimbic structures in the access and processing of dream contents. ${ }^{43}$ According to this hypothesis, emotional salience of dream imagery is due to the activation of the paralimbic cortices by the amygdala, ${ }^{87,88}$ while bizarreness of dream contents is a consequence of prefrontal deactivation, reducing the higher order control on the amygdalar and hippocampal functioning. ${ }^{110}$ It is well known that the amygdala and the hippocampal formation shows a relatively high metabolic activity during sleep. ${ }^{111,112}$ Additionally, activation of amygdala and hippocampus seems to play a pivotal role in the processing of emotional contents during REM sleep. ${ }^{113} \mathrm{On}$ the other hand, lesion studies suggested that the perceptual aspects of dreams would be related to the activation of posterior (occipital and temporal) cortices during REM sleep, and that the strong activation of high-order occipito-temporal visual cortex would explain the vivid visual imagery during dreams. ${ }^{103,104,112}$ Furthermore, the cessation of dream production is associated with posterior cortical lesions, near the temporo-occipital-parietal junction or deep bilateral frontal damage, in correspondence with the ventro-mesial region. ${ }^{11,25}$
As already stated, direct investigations on cognitive performance and dream features in adulthood have been seldom carried out. However, early studies assessed the predictability of dream recall rates in relationship with visual memory measures ${ }^{114}$ and visuospatial abilities. ${ }^{1}$ Specifically, Cory and Ormiston ${ }^{114}$ compared a group of high recallers to a group of nonrecallers, selected from a sample of college students who completed the Sleep and Dream Survey, a self-administered questionnaire about sleep characteristics and dream information. ${ }^{114}$ Then, they tested the memory for visual stimuli by using short- and long-term memory tasks, testing also for the incidental memory condition. ${ }^{114}$ Considering the number of items recalled as a measure of memory performance, they showed that recallers reported better performance for visually presented stimuli in all three conditions than nonrecallers. Finally, the authors stated that memory performances to visually administered stimuli are predictive of dream recall rates. ${ }^{114}$

Another work showed a correlation between dream recall rates after REM sleep awakenings and visuospatial abilities. ${ }^{1}$ As already seen in infancy, ${ }^{2,3,7}$ adult subjects with better performance at the Block Design Test also reported more dreams than low performers. ${ }^{1}$ Lately, Dumel et $\mathrm{al}^{115}$ aimed to assess the gain at the mirror tracing task (a visual discrimination, visuospatial motricity, and eye-hand coordination task, assumed to be REM sleep sensitive) in high and low recallers. Coherently with the expectations, the low recallers reported worst performance at the baseline in this task compared to high recallers.

Moreover, Nielsen et $\mathrm{al}^{116}$ found that spindle density typically related to cognitive functions ${ }^{117-119}$ - positively correlates with measures of dream recall (ie, dream word count) and visuospatial performance (mirror tracing test) in 53 healthy subjects. ${ }^{116}$

All these results are consistent with the previously mentioned findings of Foulkes, ${ }^{2,4}$ suggesting, again, a relationship between cognitive performance, particularly visuospatial abilities, and dreaming. ${ }^{1114-116}$

Considering the aforementioned findings, it might be interesting to have a detailed investigation on individual performance in neuropsychological tasks in relationship to dream recall frequency and dream content in adulthood. This could also open the perspective of a direct comparison with the findings available in infancy ${ }^{3,7}$ and of a continuity with an age-related cognitive decline in elderly. Nonetheless no direct investigation about brain maturation and its relationship with dream recall rates in the life span are yet available, further investigations on dream features within a neuropsychological perspective can help addressing this issue. 
Bearing in mind the already mentioned limitations in the dream research, ${ }^{21}$ it should be useful to address some final observations. Specifically, in the discussed EEG studies dream reports were collected after the first sleep cycle, ${ }^{31}$ the entire night, ${ }^{28}$ during a 40 -hour multiple nap protocol, ${ }^{101}$ diurnal naps, ${ }^{27,35}$ and REM or NREM sleep-onset periods. ${ }^{23}$ The influence of circadian and ultradian factors on dream features and recall rates ${ }^{52-57}$ could be a possible explanation for the inconsistent findings in EEG correlates of dream recall. Moreover, different time of collection of dream report (ie, in the middle of the night compared to the end or the beginning) could lead to different qualitative features in dream recall, due to a direct influence of circadian and homeostatic influencing factors. ${ }^{52-57}$

\section{Dreaming in elderly}

The relationship between sleep and memory functioning ${ }^{119-121}$ gains importance if considered in the frame of age-dependent cognitive deterioration and changes in dream recall rates. Nonetheless, only few studies investigated this issue. The question we would like to introduce is whether the reported overall reduction in dream recall frequency can result from related macro- and microstructural changes of sleep, and its relation with quantitative and qualitative features in dream content.

Sleep in elderly shows an alteration in its architecture, circadian, and homeostatic features. ${ }^{122}$ Specifically, sleep in elderly people is characterized by lower total sleep time, sleep efficiency, and SWS; a raise in intrasleep awakenings; and higher stages 1 and 2 of sleep and naps compared to younger groups. ${ }^{122}$

For what concerns REM sleep, some researchers observed a reduction in REM sleep percentage. ${ }^{122}$ Moreover, elderly people wake up more often from stage 2 than from REM sleep. ${ }^{122}$ Interestingly, K complexes ${ }^{123,124}$ and sleep spindles ${ }^{124-126}$ also decrease. These graphoelements have been addressed as markers for early cognitive deterioration conditions, such as Alzheimer's disease, ${ }^{127-129}$ where memory deterioration is one of the main complaints. Additionally, a reduced delta activity (ie, slow-wave activity) and SWS, ${ }^{56,123,130}$ conjointly with an increased beta activity, have been observed in elderly compared to younger subjects. ${ }^{57}$

Within a sleep psychophysiological perspective, Chellappa et $\mathrm{l}^{32}$ studied EEG activity and its cortical topography that is associated with a successful dream recall in the elderly. They found an NREM pattern of higher delta activity in the frontal areas at sleep offset and higher centro-parietal sigma activity in recallers compared to nonrecallers. ${ }^{32}$ This is in contrast with the findings of younger adults, where a successful dream recall was found consequently to a reduction in the delta and sigma activity. ${ }^{28,30,31,35,102}$

Despite the fact that the overall findings disclose an age-related reduction in dream recall frequency, it should be underlined that dream recall frequency seems already reduced in first and middle adulthood. ${ }^{72,132}$ Consequently, the simple relationship between aging and dream recall decline has been confuted by different studies reporting a reduction in the number of dreams remembered from around 20-38 years. ${ }^{72,132,133}$ Anyway, these results are not ever confirmed, considering a recent longitudinal study showing that dream recall rates remain stable within 3 years in $75 \%$ of the participants included in an extensive online investigation (two groups of 2872 and 2929 persons). ${ }^{143}$ Anyway, these results do not completely exclude an interpretation in terms of declined cognitive performance. Specifically, in a recent study, Nielsen ${ }^{72}$ assessed dream recall frequency and theme diversity in the life span of an individual, through an online questionnaire. The results point to sex differences in dream recall drop, with a later decline for women (which begins at 40-49 years and shows its nadir around 50-59 years) compared to men (which begins at 30-39 years and reaches its nadir at $40-49$ years). ${ }^{72}$ An additional aim was to assess the dream theme diversity in the life span throughout 56 dream themes (Typical Dreams Questionnaire [TDQ]). ${ }^{72}$ The results showed a linear decrement in the number of dream themes across ages. The author considered TDQ as a measure of episodic or voluntary autobiographical memory, interpreting the observed flattening in dream theme diversity as linked to the decline in this kind of memory in aging. ${ }^{72}$

While these results ${ }^{72}$ support only indirectly the perspective of a memory-dreaming relationship, Waterman ${ }^{131}$ tried to directly address this relationship in a systematic study with elderly people. Interestingly, quantitative features of homecollected dreams in elderly people (61-75 years) compared to middle-aged adults (45-60 years) did not show any age effect. ${ }^{131}$ More in detail, dream narrative length and frequency of dream recall showed no significant differences on either measure of recall comparing the ages of 45 and 75 years. ${ }^{131}$ At the same time, the authors found a strong positive correlation between visual memory measures and dream length, but not with dream recall frequency. ${ }^{131}$ This suggests that narrative length and recall frequency may reflect different aspects of dream recall.

Considering the paucity of studies aimed to evaluate the neural correlates of dream recall in elderly, we can only hypothesize these changes are associated to the general reduction of dream recall frequency, as reported throughout 
retrospective questionnaires. ${ }^{72,131-134}$ Some authors suggested the observed age-dependent delay in circadian rhythms could be an influencing factor on dream recall..$^{32,53,72,135,138}$ In this regard, Antrobus et $\mathrm{al}^{53}$ observed that a delay of 3 hours in sleep onset leads to longer and more vivid dream report, compared to a control group with normal sleep schedule, specifically at the end of the night. Chellappa et $\mathrm{al}^{32}$ found that elderly people have more intense and anticipated emotional load in dream content compared to a younger group.

Previous findings ${ }^{132,137}$ report that the global impoverishment of REM sleep could parallel the reduction in dream recall rates, in line with evidence of patients suffering from mild degenerative dementia who show both decreases of dream recall and REM sleep compared to healthy persons. ${ }^{138}$

In the perspective of qualitative and quantitative features of dream content, a reduction in dream salience linked to aging has been interpreted as diminished interest and overall impact associated to dream contents. ${ }^{61,132}$ This interpretation has been substantiated by an increment of dream recall rates in elderly people motivated to remember their dreams. ${ }^{139,140}$ Anyway, retrospective questionnaires - commonly used in these studies - could lead to a bias in the actual numbers of dreams remembered. ${ }^{132}$ In fact, elderly people are more susceptible to memory interference. ${ }^{141}$ Collecting dream report immediately upon awakening may partly overpass this problem. ${ }^{132,133}$ In this context, the actual finding of a reduced dream recall frequency in elderly people after provoked awakenings, as compared to younger subjects, ${ }^{131,142}$ gives strength to this interpretation in terms of age-related cognitive decline. Moreover, the motivational explanation ${ }^{139,140}$ can be interpreted in the context of a training-related gain in performance: focusing on the task of reporting dream could promote a substantial increase in salience of dream content, with a parallel improvement in memory for dreams.

Summing up, the findings available about qualitative and quantitative features of dream experience in elderly do not follow a coherent trend, leaving several opened questions about the actual involvement of an age-related cognitive decline. ${ }^{61,132}$ Moreover, the finding of a drop in dream recall rates starting from mid-adulthood ${ }^{72}$ seems counterintuitive in respect to the age-related decline hypothesis. Notwithstanding, the results of a relationship between visual memory and dream length reported by Waterman, ${ }^{131}$ as well as the indirect link between dream recall decline and declarative memory addressed by Nielsen, ${ }^{72}$ strengthen the hypothesis of parallel development of cognitive performance and qualitative/quantitative features of dream reports across the life span. However, the specificity of this relationship has still to be clarified.
In electrophysiological perspective, age-related microstructural changes of sleep have been linked to cognitive deterioration. ${ }^{123,127-129}$ Consistently, investigations in pathological cohorts could directly assess the relationship between microstructural changes and drop in dream recall rate. At the same time, in a neuropsychological perspective, specific rehabilitation programs aiming to enhance episodic-declarative performance in the elderly people may help to investigate the relationship between dream recall and cognitive performance.

\section{Conclusion and perspectives}

The aim of this review was to summarize the current investigations about sleep mentation in the life span, in the perspective to understand whether these changes are associated to an age-related mental development. In this contest, the available literature highlighted a general gap in the investigations about dream recall across the life span. The studies on neural correlates of dreaming almost completely refer to young and middle adulthood. On the other hand, very few studies directly looked for a relationship between dream content and cognitive skills. ${ }^{1,2,4,6,7,114-116}$ Consequently, definitive conclusions about dream mentation in the life span could not be addressed, either on a neural or on cognitive level.

It should be outlined that the indirect access to dream content is a stable issue in dream studies, ${ }^{21}$ nonetheless the reasons could change among ages. The variety of methods used to investigate dream mentation is probably one of the main problems that confound the interpretation in terms of basic cognitive functions. Firstly, children's studies are mainly based on reports by the caregiver. ${ }^{5,7,79}$ Furthermore, access to dream recall in children is not always possible. As an example, infantile amnesia and confabulatory reports in infancy are the main reasons why reliable studies in this age range cannot be carried out. ${ }^{73,74}$

Although for different reasons, in late ages also the study of dream mentation is challenged by a less reliable access to dream content. A decline of interests in dreaming and its emotional salience leads to a diminished access to dreaming, ${ }^{61,132}$ and consequently to a drop in dream recall rates. Moreover, a deterioration of long-term memory could be the basis for a reduced access to dream content, ${ }^{61}$ while the interference of environmental stimuli in sleep offset results in the forgetting of dream content (ie, a consolidation issue). ${ }^{132}$

Some studies on dreaming in adults focused on the hypothesis of common electrophysiological mechanisms of dream recall and wake declarative memory, aiming to overcome the limitation of an indirect access to dream mentation. ${ }^{21,27,28}$ In other words, we suggested a continuity between wake and 
sleep mental activity, hypothesizing that dream recall and episodic-declarative memory share some common mechanisms. ${ }^{21,27,28,35}$ Interestingly, brain maturational markers such as alpha and theta rhythms ${ }^{94}$ have been found to be involved both in dream recall ${ }^{21,27,28,30-32,35}$ and episodic-declarative memory ${ }^{61,94,97}$ However, this still remains an open question and the findings about this relationship are not conclusive.

Current studies do not directly address age-related memory decline as an explanation for the drop in dream recall rates. In fact, the changes in dream recall rate seem to follow a linear decrement from first adulthood to late ages. ${ }^{72}$ Executive attention and mental imagery skills have been related to dream content in children, ${ }^{3,7}$ while a positive correlation between visual memory and dream length has been reported in late ages. ${ }^{131}$ This evidence suggests the need for longitudinal investigations on specific cognitive mechanisms associated to dream experience (ie, executive functions, visuospatial ability, and episodic-declarative memory). ${ }^{3,17}$

Additionally, transversal studies should compare younger and older people with respect to both characteristics of dream recall and tasks reflecting an age-related cognitive decline. Conversely, studies in cognitive impaired cohorts, enrolled in rehabilitation protocols, should evaluate the possible covariation between changes in dreaming and the gain in cognitive performance.

Finally, our paper would provide a better understanding of the changes in dreaming during the life span as associated to an age-related mental development. However, we are aware that other factors influence sleep mentation. As an example, some studies revealed that gender differences, gender orientation, ${ }^{72,143-145}$ and personality ${ }^{146}$ also affect dream recall frequency and dream features. However, it should be underlined that the aim of this review was not to be exhaustive of the vast and complex body of literature on dreaming, but to offer a guideline for further investigations.

As a concluding remark, we would like to suggest that dreaming should be considered as a changing process, covarying and possibly paralleling mental development. Far from being a simple by-product of a sleeping mind, dreaming may also play a possible role in neural and cognitive development.

\section{Acknowledgment}

This work was supported by a grant to Serena Scarpelli from "BIAL Foundation (grant 2016/2017 number 32/16)".

\section{Disclosure}

The authors report no conflicts of interest in this work.

\section{References}

1. Butler SF, Watson R. Individual differences in memory for dreams: the role of cognitive skills. Percep Mot Skills. 1985;61(3):823-828.

2. Foulkes D. Children's Dreams: Longitudinal Studies. New York: John Wiley \& Sons Inc.; 1982.

3. Foulkes D, Hollifeld M, Sullivan B, Bradley L, Terry R. REM dreaming and cognitive skills at ages 5-8: a cross-sectional study. Int J Behav Dev. 1990;13(4):447-465.

4. Foulkes D. Dreaming and REM sleep. J Sleep Res. 1993;2:199-202.

5. Sándor P, Szakadát S, Bódizs R. Ontogeny of dreaming: a review of empirical studies. Sleep Med Rev. 2014;18(5):435-449.

6. Sándor P, Szakadát S, Kertész K, Bódizs R. Content analysis of 4 to 8 years-old children's dream reports. Front Psychol. 2015;6:534.

7. Sándor P, Szakadát S, Bódizs R. The development of cognitive and emotional processing as reflected in children's dreams: active self in an eventful dream signals better neuropsychological skills. Dreaming. 2016;26(1):58-78.

8. Bell A, Hall C. The Personality of a Child Molester: An Analysis of Dreams. Chicago, IL: Aldine; 1971.

9. Hall C. A cognitive theory of dreams. J Gen Psychol. 1953;49(1): 273-282.

10. Domhoff GW. The neural substrate for dreaming: is it a subsystem of the default network? Conscious Cogn. 2011;20:1163-1174.

11. Domhoff GW. The invasion of the concept snatchers: he origins, distortions, and future of the continuity hypothesis. Dreaming. 2017;27(1):14-39.

12. Hobson JA, Schredl M. The continuity and dis-continuity between waking and dreaming: a Dialogue between Michael Schredl and Allan Hobson concerning the adequacy and completeness of these notions. Int J Dream Res. 2011;4:3-7.

13. Ross JH. The continuity and discontinuity between waking and dreaming from the perspective of an analytical psychological construct. Commentary on "The continuity and discontinuity between waking and dreaming: A Dialogue between Michael Schredl and Allan Hobson concerning the adequacy and completeness of these notions". Int $J$ Dream Res. 2011;4(2):81.

14. Schredl M. Continuity in studying the continuity hypothesis of dreaming is needed reply to the comments on "The continuity and discontinuity between waking and dreaming: A dialogue between Michael Schredl and Allan Hobson concerning the adequacy and completeness of these notions". Int J Dream Res. 2012;5(1):1-8.

15. De Gennaro L, Marzano C, Cipolli C, Ferrara M. How we remember the stuff that dreams are made of: neurobiological approaches to the brain mechanisms of dream recall. Behav Brain Res. 2012;226:592e6.

16. Cipolli C, Ferrara M, De Gennaro L, Plazzi G. Beyond the neuropsychology of dreaming: insights into the neural basis of dreaming with new techniques of sleep recording and analysis. Sleep Med Rev. 2017;35:8-20.

17. Aserinsky E, Kleitman N. Regularly occurring periods of eye motility, and concomitant phenomena, during sleep. Science. 1953;118:273-274

18. Dement, Kleitman N. The relation of eye movements during sleep to dream activity: an objective method for the study of dreaming. $J$ Exp Psychol. 1957;53:339-346.

19. Dement W, Kleitman N. Cyclic variations in EEG during sleep and their relation to eye movements, body motility, and dreaming. Electroencephalogr Clin Neurophysiol. 1957;9(4):673-690.

20. Foulkes D. Dream reports from different stages of sleep. J Abnorm Soc Psychol. 1962;65:14-25.

21. Scarpelli S, D'Atri A, Gorgoni M, Ferrara M, De Gennaro L. EEG oscillations during sleep and dream recall: state- or trait-like individual differences? Front Psychol. 2015; 6:605.

22. Nielsen T. A review of mentation in REM and NREM sleep: covert REM sleep as a possible reconciliation of two opposing models. Behav Brain Sci. 2000;23:851-866. 
23. Hobson JA, McCarley RW. The brain as a dream state generator: an activation-synthesis hypothesis of the dream process. Am J Psychiat. 1977;134:1335-1348.

24. Foulkes D. Dreaming: A Cognitive-Psychological Analysis. Hillsdale, NJ: Erlbaum; 1985.

25. Solms M. The Neuropsychology of Dreams: A Clinico-Anatomical Study. Hillsdale: Erlbaum; 1997.

26. Solms M. Dreaming and REM sleep are controlled by different brain mechanisms. Behav Brain Sci. 2000;2:843-850.

27. Scarpelli S, Marzano C, D’Atri A, Gorgoni M, Ferrara M, De Gennaro L. State-or trait-like individual differences in dream recall: preliminary findings from a within-subjects study of multiple nap REM sleep awakenings. Front Psychol. 2015;6:928.

28. Marzano C, Ferrara M, Mauro F, et al. Recalling and forgetting dreams: theta and alpha oscillations during sleep predict subsequent dream recall. J Neurosci. 2011;31:6674-6683.

29. Hsieh LT, Ranganath C. Frontal midline theta oscillations during working memory maintenance and episodic encoding and retrieval. Neuroimage. 2014;85:721-729.

30. Takeuchi T, Ogilvie RD, Murphy TI, Ferrelli AV. EEG activities during elicited sleep onset REM and NREM periods reflect different mechanisms of dream generation. Clin Neurophysiol. 2003;114:210-220.

31. Esposito MJ, Nielsen TA, Paquette T. Reduced alpha power associated with the recall of mentation from Stage 2 and Stage REM sleep. Psychophysiology. 2004;41:288-297.

32. Chellappa SL, Münch M, Blatter K, Knoblauch V, Cajochen C. Does the circadian modulation of dream recall modify with age? Sleep. 2009;32:1201.

33. Nyhus E, Curran T. Functional role of gamma and theta oscillations in episodic memory. Neurosci Biobehav Rev. 2010;34:1023-1035.

34. Corsi-Cabrera M, Miro E, del-R1o-Portilla Y, Perez-Garci E, Villanueva Y, Guevara MA. Rapid eye movement sleep dreaming is characterized by uncoupled EEG activity between frontal and perceptual cortical regions. Brain Cogn. 2002;51:337-345.

35. Scarpelli S, D'Atri A, Mangiaruga A, et al. Predicting dream recall: EEG activation during NREM sleep or shared mechanism with wakefulness? Brain Topogr. 2017;30(5):629-638.

36. Bartolacci C, Scarpelli S, De Gennaro L. L'attività elettrica cerebrale (EEG) predice la presenza del ricordo dei sogni? Riv Sperim Freniatria. 2017;2:79-99.

37. Schredl M. Questionnaires and diaries as research instruments in dream research: methodological issues. Dreaming. 2002;12:17-26.

38. Schredl M. Continuity between waking and dreaming: a proposal for a mathematical model. Sleep Hypnosis. 2003;5:26-40.

39. Robert G, Zadra A. Measuring nightmare and bad dream frequency: impact of retrospective and prospective instruments. J Sleep Res. 2008; 17:132-139.

40. Levin R, Nielsen TA. Disturbed dreaming, posttraumatic stress disorder, and affect distress: a review and neurocognitive model. Pych Bull. 2007;133:482-528.

41. Schredl M, Wittmann L. Dreaming: a psychological view. Schweiz Arch Neurol Psychiatr. 2005;156:484-492.

42. Schwartz S, Maquet P. Sleep imaging and the neuropsychological assessment of dreams. Trends Cogn. 2002;6:23-30.

43. Hobson JA, Pace-Schott EF, Stickgold R. Dreaming and the brain: toward a cognitive neuroscience of conscious states. Behav Brain Sci. 2000;23:793-842.

44. Hall CS, Van de Castle RL. The Content Analysis of Dreams. New York: Appleton-Century-Crofts; 1966.

45. Domhoff GW. New directions in the study of dream content using the Hall and Van de Castle coding system. Dreaming. 1999;9(2-3):115.

46. Nielsen TA. Dream analysis and classification: the reality simulation perspective. In: Kryeger M, Roth T, Dement WC, editors. Principles and Practice of Sleep Medicine. New York: Elsevier; 2010:595-603.

47. Schredl M. Dream content analysis: basic principles. Int J Dream Res. 2010;3(1):65-73.
48. Van Rijn E, Eichenlaub JB, Lewis PA, et al. The dream-lag effect: selective processing of personally significant events during rapid eye movement sleep, but not during slow wave sleep. Neurobiol Learn Mem. 2015;122:98-109.

49. Blagrove M, Henley-Einion J, Barnett A, Edwards D, Seage CH. A replication of the 5-7day dream-lag effect with comparison of dreams to future events as control for baseline matching. Conscious Cogn. 2011;20(2):384-391.

50. Blagrove M, Fouquet NC, Henley-Einion JA, et al. Assessing the dream-lag effect for REM and NREM Stage 2 dreams. PLoS One. 2011;6(10):e26708.

51. Vallat R, Chatard B, Blagrove M, Ruby P. Characteristics of the memory sources of dreams: a new version of the content-matching paradigm to take mundane and remote memories into account. PLoS One. 2017;12(10):e0185262.

52. Antrobus JS. Dreaming: cognitive processes during cortical activation and high afferent thresholds. Psychol Rev. 1991;98:95-121.

53. Antrobus, J, Kondo T, Reinsel R, Fein G. Dreaming in the late morning: summation of REM and diurnal cortical activation. Conscious Cogn. 1995;4:275-299.

54. Wamsley EJ, Hirota Y, Tucker MA, Smith MR, Antrobus JS. Circadian and ultradian influences on dreaming: a dual rhythm model. Brain Res Bull. 2007;71(4):347-354.

55. Chellappa SL, Cajochen C. Ultradian and circadian modulation of dream recall: EEG correlates and age effects. Int J Psychophysiol. 2013;89(2):165-170.

56. Cajochen C, Münch M, Knoblauch V, Blatter K, Wirz-Justice A. Agerelated changes in the circadian and homeostatic regulation of human sleep. Chronobiol Int. 2006;23:461-474.

57. Münch M, Silva EJ, Ronda JM, Czeisler CA, Duffy JF. EEG sleep spectra in older adults across all circadian phases during NREM sleep. Sleep. 2010;33(3):389-401.

58. Siclari F, LaRocque JJ, Postle BR, Tononi G. Assessing sleep consciousness within subjects using a serial awakening paradigm. Front Psychol. 2013;4:542.

59. Siclari F, LaRocque JJ, Bernardi G, Postle BR, Tononi G. The neural correlates of consciousness in sleep: a no-task, within-state paradigm. bioRxiv. Epub December 2014.

60. Weisz R, Foulkes D. Home and laboratory dreams collected under uniform sampling conditions. Psychophysiology. 1970;6:588-596.

61. Cohen DB. Remembering and forgetting dreaming. In: Kihlstrom JF, Evans FJ, editors. Functional Disorders of Memory. NewYork: Wiley; 1979: 239-274.

62. Nielsen T. Chronobiological features of dream production. Sleep Med Rev. 2004;8(5):403-424.

63. Snyder F. The phenomenology of dreaming. In: Meadow L, Snow L, editors. The Psychodynamic Implications of the Physiological Studies on Dreams. Springfield: Thomas; 1970:124-151.

64. Foulkes D, Pope R. Primary visual experience and secondary cognitive elaboration in stage REM: a modest confirmation and an extension. Percept Mot Skills. 1973;37:107-118.

65. Foulkes D, Schmidt M. Temporal sequence and unit composition in dream reports from different stages of sleep. Sleep. 1983;6:265-280.

66. Wittmann L, Zehnder D, Schredl M, Jenni OG, Landolt MA. Posttraumatic nightmares and psychopathology in children after road traffic accidents. J Trauma Stress. 2010;23:232e9.

67. Simard V, Nielsen, T, Tremblay RE, Boivin M, Montplaisir JY. Longitudinal study of preschool sleep disturbance. The predictive role of maladaptive parental behaviors, early sleep problems, and child/ mother psychological factors. Arch Pediatr Adolesc Med. 2008;162:4.

68. Simard V, Nielsen TA, Tremblay RE, Boivin M, Montplaisir JY. Longitudinal study of bad dreams in preschool-aged children: prevalence, demographic correlates, risk and protective factors. Sleep. 2008;31(1):62-70.

69. Siegel AB. Children's dreams and nightmares: emerging trends in research. Dreaming. 2005;15(3):147-154. 
70. Germain A, Nielsen TA. Sleep pathophysiology in posttraumatic stress disorder and idiopathic nightmare sufferers. Biol Psychiatry. 2003;54(10):1092-1098.

71. Schredl M. Home dream recall in children and young adults. Int $J$ Dream Res. 2009;2(2):58-59.

72. Nielsen T. Variations in dream recall frequency and dream theme diversity by age and sex. Front Neurol. 2012;4(3):106.

73. Nir Y, Tononi G. Dreaming and the brain: from phenomenology to neurophysiology. Trends Cogn Sci. 2010;14:88-100.

74. Llewellyn S. Such stuff as dreams are made on? Elaborative encoding, the ancient art of memory, and the hippocampus. Behav Brain Sci. 2013;36:589-659.

75. Foulkes D. Home and laboratory dreams: four empirical studies and a conceptual reevaluation. Sleep. 1979;2:233e51.

76. Wechsler D. Manual for the Wechsler Preschool and Primary Scale of Intelligence. San Antonio: The Psychological Corporation; 1967.

77. Groth-Marnat G, Teal M. Block design as a measure of everyday spatial ability: a study of ecological validity. Percept Mot Skills. 2000;90(2):522-526.

78. Voss U, Frenzel C, Koppehele-Gossel J, Hobson A. Lucid dreaming: an age-dependent brain dissociation. J Sleep Res. 2012;21(6): 634-642.

79. Colace C. Children's Dreams: From Freud's Observations to Modern Dream Research. 1st ed. London: Karnac Books Ltd; 2010.

80. Valli K, Revonsuo A, Pälkäs O, Ismail KH, Ali KJ, Punamäki R-L. The threat simulation theory of the evolutionary function of dreaming: evidence from dreams of traumatized children. Conscious Cogn. 2005; $14: 188 \mathrm{e} 218$.

81. Duke T, Davidson J. Ordinary and recurrent dream recall of active, past and non-recurrent dreamers during and after academic stress. Dreaming. 2002;12:185-197.

82. Punamaki RL. Determinants and mental health effects of dream recall among children living in traumatic conditions. Dreaming. 1997; 7:235-263.

83. Terr LC. Children of Chowchilla: a study of psychic trauma. Psychoanal Study Child. 1979;34:547e623.

84. Helminen E, Punamäki RL. Contextualized emotional images in children's dreams: psychological adjustment in conditions of military trauma. Int J Behav Dev. 2008;32:177-187.

85. De Gennaro L, Ferrara M, Cristiani R, Curcio G, Martiradonna V, Bertini M. Alexithymia and dream recall upon spontaneous morning awakening. Psychosom Med. 2003;65:301-306.

86. De Gennaro L, Marzano C, Moroni F, Curcio G, Ferrara M, Cipolli C. Recovery sleep after sleep deprivation almost completely abolishes dream recall. Behav Brain Res. 2010;206:293-298.

87. De Gennaro L, Cipolli C, Cherubini A, et al. Amygdala and hippocampus volumetry and diffusivity in relation to dreaming. Hum Brain Mapp. 2011;32:1458-1470.

88. De Gennaro L, Lanteri O, Piras F, et al. Dopaminergic system and dream recall: an MRI study in Parkinson's disease patients. Human Brain Mapp. 2016;37:1136-1147.

89. Strauch I. REM dreaming in the transition from late childhood to adolescence: a longitudinal study. Dreaming. 2005;15:155e69.

90. Evans RC. Dream conception and reality testing in children. J Am Acad Child Psychiatr. 1973;12(1):73-92.

91. Strauch I, Lederbogen S. The home dreams and waking fantasies of boys and girls between ages 9 and 15: a longitudinal study. Dreaming. 1999;9(2-3): 153.

92. Cicogna PC, Occhionero M, Natale V, Esposito MJ. Bizarreness of size and shape in dream images. Conscious Cogn. 2007;16: 381-390.

93. Nielsen T. The stress acceleration hypothesis of nightmares. Front Neurol. 2017;8:201.

94. Klimesch W. EEG alpha and theta oscillations reflect cognitive and memory performance: a review and analysis. Brain Res Rev. 1999;29:169-195.
95. Novelli L, D’atri A, Marzano C, et al. Mapping changes in cortical activity during sleep in the first 4 years of life. J Sleep Res. 2016;25(4):381-389.

96. Nishida M, Pearsall J, Buckner RL, Walker MP. REM sleep, prefrontal theta, and the consolidation of human emotional memory. Cereb Cortex. 2009; 19:1158-1166.

97. Klimesch W, Doppelmayr M, Russegger H, Pachinger T. Theta band power in the human scalp EEG and the encoding of new information. Neuroreport. 1996;7:1235-1240.

98. Anderson KL, Rajagovindan R, Ghacibeh GA, Meador KJ, Ding M. Theta oscillations mediate interaction between prefrontal cortex and medial temporal lobe in human memory. Cereb Cortex. 2010;20:1604-1612.

99. Norman KA, Newman EL, Detre G. A neural network model of retrieval-induced forgetting. Psychol Rev. 2007;114:887-953.

100. Siclari F, Baird B, Perogamvros L, et al. The neural correlates of dreaming. Nat Neurosci. 2017;20(6):872-878.

101. Chellappa SL, Frey S, Knoblauch V, Cajochen C. Cortical activation patterns herald successful dream recall after NREM and REM sleep. Biol Psychol. 2011;87(2):251-256.

102. Schredl M, Schäfer G, Weber B, Heuser I. Dreaming and insomnia: dream recall and dream content in patients with insomnia. J Sleep Res. 1998;7(3):191-198.

103. Eichenlaub JB, Nicolas A, Daltrozzo J, et al. Resting brain activity varies with dream recall frequency between subjects. Neuropsychopharmacology. 2014;39:1594-1602.

104. Eichenlaub JB, Bertrand O, Morlet D, Ruby P. Brain reactivity differentiates subjects with high and low dream recall frequencies during both sleep and wakefulness. Cereb Cortex. 2014;24: 1206-1215.

105. Voss U, Holzmann R, Hobson A, et al. Induction of self-awareness in dreams through frontal low current stimulation of gamma activity. Nat Neurosci. 2014;17:810-812.

106. Zimmerman WB. Sleep mentation and auditory awakening thresholds. Psychophysiology. 1970;6:540-549.

107. Koulack D, Goodenough DR. Dream recall and dream recall failure: an arousal-retrieval model. Psychol Bull. 1976;83:975.

108. Rosenblatt SI, Antrobus JI, Zimler JP. The effect of post-awakening differences in activation on the REM-NREM report effect and recall of information from films. In: Antrobus JS, Bertini M, editors. The Neuropsychology of Sleep and Dreaming. Hillsdale, NJ: Erlbaum; 1992.

109. Colace C. Dream bizarreness reconsidered. Sleep Hypn. 2003;5(3): $105-128$

110. Maquet P, Péters JM, Aerts J, et al. Functional neuroanatomy of human rapid eye movement sleep and dreaming. Nature. 1996;383:163-166.

111. Nofzinger EA, Mintun MA, Wiseman M, Kupfer DJ, Moore RY. Forebrain activation in REM sleep: an FDG PET study. Brain Res. 1997;770:192-201.

112. Braun AR, Balkin TJ, Wesensten NJ, et al. Regional cerebral blood flow throughout the sleep-wake cycle: an $\mathrm{H} 2 \mathrm{O}-15$ positron emission tomography study. Brain. 1997;120:1173-1197.

113. Hobson JA, Pace-Schott EF. The cognitive neuroscience of sleep: neuronal system, consciousness and learning. Nat Rev Neurosci. 2002;3(9):679-693.

114. Cory TL, Ormiston DW. Predicting the frequency of dream recall. J Abnorm Psychol. 1975;84(3):261-266.

115. Dumel G, Carr M, Marquis LP, Blanchette-Carriere C, Paquette T, Nielsen T. Infrequent dream recall associated with low performance but high overnight improvement on mirror-tracing. J Sleep Res. 2015; 24:372-382.

116. Nielsen T, Carr M, Blanchette-Carrière C, et al. NREM sleep spindles are associated with dream recall sleep spindles \& cortical up states. 2016;1(1):27-41.

117. Fogel SM, Smith CT. The function of the sleep spindle: a physiological index of intelligence and a mechanism for sleep-dependent memory consolidation. Neurosci Biobehav Rev. 2011;35(5):1154-1165. 
118. Clemens Z, Fabo D, Halasz P. Twenty-four hours retention of visuospatial memory correlates with the number of parietal sleep spindles. Neurosci Letters. 2006;403(1-2):52-56.

119. Diekelmann S, Born J. The memory function of sleep. Nat Rev Neurosci. 2010;11(2):114-126.

120. Ellenbogen JM1, Payne JD, Stickgold R. The role of sleep in declarative memory consolidation: passive, permissive, active or none? Curr Opin Neurobiol. 2006;16(6):716-722.

121. Walker MP, Stickgold R. Overnight alchemy: sleep-dependent memory evolution. Nat Rev Neurosci. 2010;11(3):218; author reply 218.

122. Pace-Schott EF, Spencer RM. Sleep-dependent memory consolidation in healthy aging and mild cognitive impairment. Curr Top Behav Neurosci. 2015;25:307-330.

123. Schwarz JFA, Akerstedt T, Lindberg E, Gruber G, Fischer H, TheorellHaglo WJ. Age affects sleep microstructure more than sleep macrostructure. J Sleep Res. 2017;26:277-287.

124. Crowley K, Trinder J, Kim Y, Carrington M, Colrain IM. The effects of normal aging on sleep spindle and K-complex production. Clin Neurophysiol. 2002;113:1615-1622.

125. Landolt HP, Dijk DJ, Achermann P, Borbély AA. Effect of age on the sleep EEG: slow-wave activity and spindle frequency activity in young and middle- aged men. Brain Res. 1996;738:205-212.

126. Carrier J, Monk TH, Buysse DJ, Kupfer DJ. Sleep and morningnesseveningness in the "middle" years of life (20-59 y). J Sleep Res. 1997;6:230-237.

127. De Gennaro L, Gorgoni M, Reda F, et al. The fall of sleep K-complex in Alzheimer disease. Sci Rep. 2017;7:39688.

128. Reda F, Gorgoni M, Lauri G, et al. In search of sleep biomarkers of Alzheimer's disease: K-complexes do not discriminate between patients with mild cognitive impairment and healthy controls. Brain Sci. 2017;7(5). pii: E51.

129. Gorgoni M, Lauri G, Truglia I, et al. Parietal fast sleep spindle density decrease in Alzheimer's disease and amnesic mild cognitive impairment. Neural Plast. 2016;2016:8376108

130. Bliwise DL. Normal aging. Princ Pract Sleep Med. 2005;4:24-38.

131. Waterman D. Aging and memory for dreams. Percept Mot Skills. 1991;73:355-365.

132. Giambra LM, Jung RE, Grodsky A. Age changes in dream recall in adulthood. Dreaming. 1996;6:17.
133. Funkhouser AT, Hirsbrunner HP, Cornu C, Bahro M. Dreams and dreaming among the elderly: an overview. Aging Ment Health. 1999;3:10-20.

134. Zanasi M, De Persis S, Caporali M, Siracusano A. Dreams and age. Percept Mot Skills. 2005;100:925-938.

135. Suzuki H, Uchiyama M, Tagaya $H$, et al. Dreaming during non-rapid eye movement sleep in the absence of prior rapid eye movement sleep. Sleep. 2004;27:1486-1490.

136. Chellappa SL, Muench M, Knoblauch V, Cajochen C. Age effects on spectral electroencephalogram activity prior to dream recall. J Sleep Res. 2012;21: 247-256.

137. Woodruff DS. Arousal, sleep, and aging. In: Birren JE, Schaie W, Bengtson V, Jarvik L, Salthouse T, editors. Handbook of the Psychology of Aging; 1985:261-295.

138. Guenole F, Marcaggi G, Baleyte JM, Garma, L. Dreams in normal and pathological aging. Psychol Neuropsychiatr Vieil. 2010;8: 87-96.

139. Strunz F. Träumen im Alter: Bestand und Nutzung [Dreams in the elderly: contents and clinical usefulness]. Gesundheitswesen. 1993;55(11): 595-601. German.

140. Strunz F. Die Kultivierung der Traumerinnerung-Ein Instrument [The cultivation of dream recall: a tool]. Psychischer Hygiene Pravention. 1988;11:67-70. German.

141. Kausler D. Experimental Psychology, Cognition, and Human Aging. 2nd ed. New York: Springer-Verlag; 1991.

142. Fein G, Feinberg I, Insel TR, et al. Sleep mentation in the elderly. Psychophysiology. 1985;22:218-225.

143. Schredl M, Göritz AS. Changes in dream recall frequency, nightmare frequency, and lucid dream frequency over a 3-year period. Dreaming. 2015;25(2):81-87.

144. Schredl M, Göritz AS. Gender differences in dream content: related to biological sex or sex role orientation? Imagination, cognition and personality. 2010; 30(2) 171-183.

145. Georgi M, Schredl M, Henley-Einion J, Blagrgove M. Gender differences in dreaming in childhood and adolescence: The UK Library study. Int J of Dream Res. 2012;5(2);125-129.

146. Malinowski JE. Dreaming and personality: wake-dream continuity, thought suppression, and the Big Five Inventory. Conscious Cogn. 2015;15(38):9-15.
Nature and Science of Sleep

\section{Publish your work in this journal}

Nature and Science of Sleep is an international, peer-reviewed, open access journal covering all aspects of sleep science and sleep medicine including the neurophysiology and functions of sleep, the genetics of sleep, sleep and society, biological rhythms, dreaming, sleep disorders and therapy, and strategies to optimize healthy sleep. The manuscript

\section{Dovepress}

management system is completely online and includes a very quick and fair peer-review system, which is all easy to use. Visit http://www. dovepress.com/testimonials.php to read real quotes from published authors. 